\title{
An Efficient Hybrid Model and Dynamic Performance Analysis for Multihop Wireless Networks
}

\author{
Kunjie $\mathrm{Xu}^{\dagger}$, David Tipper ${ }^{\dagger}$, Prashant Krishnamurthy ${ }^{\dagger}$, Yi Qian* \\ Graduate Telecommunications and Networking Program, University of Pittsburgh ${ }^{\dagger}$ \\ Department of Computer and Electronics Engineering, University of Nebraska-Lincoln* \\ Emails: kux1@pitt.edu,dtipper@pitt.edu,prashant@sis.pitt.edu,yqian2@unl.edu
}

\begin{abstract}
Multihop wireless networks are frequently subjected to nonstationary phenomena due to dynamic network topology. However, the discrete event simulation normally uses the steadystate statistical analysis to study the performance of this type of networks, even though transient or nonstationary period will occur often and likely dominant the network behavior. Moreover, the majority of the simulators suffer from scalability issue. In this paper, we develop an efficient performance modeling technique for analyzing the time varying performance of multihop wireless networks. The one-hop packet transmission (service) time is assumed to be deterministic, which could be achieved by contention-free transmission, or generally approximated in sparse or lightly loaded multihop wireless network. Our model is a hybrid of time varying adjacency matrix and fluid flow based differential equations, which represent dynamic topology changes and nonstationary network queues, respectively. Numerical experiments show that the hybrid fluid based model can provide reasonably accurate results much more efficiently than standard simulator in terms of the computational time. Furthermore, the network performance is extensively studied via hybrid model by considering the impact from node mobility, traffic load and wireless link quality.
\end{abstract}

\section{INTRODUCTION}

In recent years, there has been significant growth of interest in multihop wireless networks, such as wireless mesh networks (WMN) [1], vehicular ad-hoc networks (VANET) [2], wireless sensor networks (WSN) [3] and mobile ad-hoc networks (MANET) [4]. Multihop wireless networks are expected to become an important component of the communications landscape and may work in a fully autonomous scenario or as an extension to an infrastructure network. In multihop wireless networks, each node participates in routing by forwarding data for other nodes due to their limited radio communication range. The determination of which nodes forward data is made dynamically based on the network topology. Since the network nodes may have the ability to move, the network topology is expected to change often and unpredictably. Meanwhile, multihop wireless networks also inherit the traditional problems of communications over wireless channels. These problems combined together make it challenging to accurately evaluate and predict the performance of multihop wireless networks.

This research was funded in part by the US Army Research Office under the Multi-University Research Initiative (MURI) grant W911NF-07-1-0318.
The performance of multihop wireless networks is normally studied via simulation over a fixed time horizon using a steadystate type of statistical analysis procedure [5]. However, due to the dynamic network topology or nonstationary traffic, such an approach may be inappropriate as the network may spend most time in a transient/stationary state [6]. To study nonstationary behavior, the measurements of quantities observed over small intervals or at specific points in time are important. Therefore, instead of the time average in steady-state simulation, ensemble averages based nonstationary simulation are more appropriate [7]. The idea is to construct ensemble average curves of quantities of interest across a set of statistically identical but distinct independent simulation runs, along with the confidence interval. With many such points collected at different time instants, the system behavior can be shown as a function of time. However, to assure the accurate portrayal of the actual system, a large number of runs are required resulting in large amounts of CPU time and scalability issues.

The analytical model used in network performance evaluation mainly deals with the steady state conditions of queuing theory models [8]. However, to model the realistic dynamics of time-varying behavior, we have developed an approximate fluid flow modeling approach which can be used to efficiently model the mean transient/nonstationary behavior in different large complex networks of queues [9]-[11]. The method has been termed the Pointwise Stationary Fluid Flow Approximation (PSFFA) [9]. In addition, other fluid flow methods have been proposed for constructing computationally efficient models. Kim and Hou in [12] developed a fluid flow based simulator for WLAN with the consideration of the characteristics of IEEE 802.11 protocol behavior, and examined fluid simulation performance in terms of events generated, execution time required, relative error incurred, and time step value adopted in the simulation. In [13], a fluid flow model is presented to analyze the performance of backlog-based CSMA policies in a wireless network environment with multiple traffic arrival streams. While all of these approaches are related to our work, little fluid-flow related work has modeled and analyzed the performance of multihop wireless networks by considering their unique characteristics (e.g. wireless links quality, mobility, dynamic routing, and scalability). 
Our contribution in this paper can be summarized as follows. First of all, we propose a time varying performance model of multihop wireless networks with deterministic service time. As an important aspect of QoS, predictable response time is required in many on-demand or real-time networking services. In multihop wireless networks, one-hop deterministic packet transmission (service) times can be achieved by contention-free transmission with the technique of distributed scheduling [14] or service differentiation [15]. Deterministic packet transmission (service) times could also approximately occur in contention-based transmission networks which are sparse or lightly loaded. For example, energy conserving techniques in WSNs put most of the nodes in sleep modes so that the network becomes sparse. Moreover, each sensor node only has light traffic to transmit in order to to save energy. Secondary, we use the hybrid model to extensively analyze the network performance impacted by unique features of multihop wireless networks, such as stochastic node mobility and probabilistic link connectivity. Our hybrid model is shown to be an efficient, scalable and flexible tool to evaluate the time varying network performance.

The rest of this paper is organized as follows. Section II describes the details of the hybrid model. Section III shows numerical results to validates the accuracy of hybrid model by nonstationary simulation and then evaluates the scalability of the model. Section IV presents extensive experiments by using hybrid model to examine the impacts of node mobility, traffic load and link quality to network performance. Our conclusion and future work are given in Section V.

\section{Modeling Dynamic Behavior of Multihop WIRELESS NETWORKS}

\section{A. Network Topology Modeling}

Consider a multihop wireless network consisting of $M$ nodes, the network topology in terms of connectivity at any time $t$ is modeled with a $M \times M$ adjacency matrix denoted as $\mathbf{A}(t)=\left(a_{i j}(t)\right)$. Here, $a_{i j}(t)$ represents the binary link connectivity between node $i$ and $j$ (i.e., $a_{i j}(t)=1$ if link from node $i$ to $j$ exists, otherwise $a_{i j}(t)=0$ ). The link connectivity $a_{i j}(t)$ between two nodes depends on their radio range. With the assumption that all radios have a perfect coverage on a two-dimensional space, the problem of link connectivity is simplified by judging whether the distance $d_{i j}$ between node $i$ and node $j$ is within the circular coverage range (i.e., if $d_{i j} \leq R$ at time $t, a_{i j}(t)=1$; otherwise $a_{i j}=0$ ). However, it is widely understood that the actual radio link connectivity differs from this simple model due to interference and physical layer propagation error including signal attenuation, shadowing and fading [16]. Even though two nodes are in the radio range of each other, they cannot always hear each other without any data loss, and the bit error rate is typically a function of the signal to noise plus interference ratio. In order to represent real link quality as well as connectivity, we let $a_{i j}$ be a real number between 0 and 1 (i.e., $a_{i j}(t)=p \in(0,1]$ if link from node $i$ to $j$ exists, otherwise $a_{i j}(t)=0$ ).
To model the node mobility, our approach is to directly manipulating the elements of the adjacency matrix according to a planned experiment (as in Section III) or a stochastic/probabilistic model (as in Section IV). Such a probabilistic model can be developed either from the mobility model assumptions and analysis [17] or from fitting a statistical model to data gathered from a test bed or simulation (e.g., twostate MMPP [18]). The probabilistic link connectivity model was proposed with original intention to solve the shortcomings of long warm up period and high computational requirement needed in any discrete event wireless multihop simulation, which computes node position based on geography of the simulated space and determines the link connection between each node pair via wireless propagation models.

\section{B. Fluid Flow Model Background}

In developing a performance model of the network, we start with modeling a single queue and then generalize to a arbitrary queue in a network. To describe the time varying behavior of the queue at each network node, we adopt the concept of Pointwise Stationary Fluid Flow Approximation (PSFFA). To understand this fluid flow approach, we first give a description of the general background.

For the scenario of a FIFO queueing system with nonstationary arrival process, we define $x(t)$ as the state variable representing the ensemble average number of packet in the system at time $t$. Let $\dot{x}(t)=d x / d t$ be the change rate of the state variable with respect to time. Following the flow conservation principle, we have the change rate of the average packet number in the system $\dot{x}(t)$ equals to the difference between the flow in $f_{\text {in }}(t)$ and the flow out $f_{\text {out }}(t)$ of the system at time $t$ as:

$$
\dot{x}(t)=-f_{\text {out }}(t)+f_{\text {in }}(t)
$$

For the infinite-size queue without packet dropping, we simply have $f_{\text {in }}(t)=\lambda(t)$, where $\lambda(t)$ represents the ensemble average arrival rate at time $t$. The flow out can be related to the average utilization of the server as $f_{\text {out }}(t)=\mu C G(x(t))$, where $1 / \mu$ refers to the average packet length (bits), and $C$ defines the server capacity (link bandwidth, bps). Then $\mu C$ denotes the average service rate (pkt/s). The average link utilization $G(x(t))$ is a monotonically increasing function of $x(t)$. This function is defined in the range $G(x) \in[0,1)$ and passes through the origin $G(0)=0$. Hence, the fluid flow equation (1) can be written as:

$$
\dot{x}(t)=-\mu C G(x(t))+\lambda(t)
$$

Equation 2 is quite general and can model a wide range of queueing systems [10]. The utilization function $G(x(t))$ is determined by the stochastic modeling of the queue such as traffic arrival process and service time distribution.

\section{Multiclass Queue with Deterministic Service Times}

In general, the deterministic service times are practical and frequently applied in telecommunication system. The idea of fixed length packets facilitates network design especially 
when dealing with congestion control and fairness issues. In this work, we consider the multihop wireless networks with deterministic service time and Poisson traffic arrivals at each node. Hence, the statistical behavior of each node is modeled as $M / D / 1$ queue. From queueing theory, the average packet number in the system at steady state is given by $x(t)=$ $\rho+\rho^{2} /(2(1-\rho))$, where $\rho=G(x(t))=\lambda(t) /(\mu C)$. We apply the PSFFA technique matching the stead-state equilibrium points to obtain the utilization function $G(x(t))$. As a result, under steady state conditions, (i.e., $\dot{x}(t)=0$ ), the state model turns out to be:

$$
\dot{x}(t)=-\mu C\left(x(t)+1-\sqrt{x(t)^{2}+1}\right)+\lambda(t)
$$

In multihop wireless networks, the traffic in the network is normally divided into a number of classes and the control actions (i.e. routing and flow control) are based on the class type. Thus we extend the fluid flow model into multiclass traffic case. For the queue with $S$ classes traffic, $x_{l}(t)$, and $\lambda_{l}(t)$ represent the ensemble average number of packet and arrival rate of class $l$ traffic, while $x_{T}=\sum_{l=1}^{S} x_{i}$ and $\lambda_{T}=$ $\sum_{l=1}^{S} \lambda_{i}$ denote the total ensemble average number of packet and the mean aggregate arrival rate into the system. Then, the fluid flow model in (2) becomes:

$$
\dot{x}_{T}(t)=-\mu C\left(x_{T}(t)+1-\sqrt{x_{T}(t)^{2}+1}\right)+\lambda_{T}(t)
$$

The flow conservation principle still applies to each traffic class; therefore, a fluid flow model in (2) can also be developed for each class with $G\left(x_{l}(t), x_{T}(t)\right)$ as the average utilization function of class $l$ traffic in the multiclass queue.

$$
\begin{array}{r}
\dot{x}_{l}(t)=-\mu C G_{l}\left(x_{l}(t), x_{T}(t)\right)+\lambda_{l}(t) \\
\forall l=1,2, \ldots, S
\end{array}
$$

Note that at steady state, the average number of total packets in the $M / D / 1$ queuing system is

$$
x_{T}(t)=\frac{\lambda_{T}}{\mu C}+\frac{\lambda_{T}^{2}}{2 \mu^{2} C^{2}\left(1-\frac{\lambda_{T}}{\mu C}\right)}
$$

From multiclass queuing theory [8], we can write the steady-state number of packets of class $l$ traffic $x_{l}(t)$ as

$$
x_{l}(t)=\frac{\lambda_{l}\left(2 \mu C-\lambda_{T}\right)}{2 \mu C\left(\mu C-\lambda_{T}\right)}
$$

Following the approach of steady state equilibrium matching with $\dot{x}_{T}(t)=0$ and $\dot{x}_{l}(t)=0$, we get $\lambda_{T}(t)=$ $\mu C\left(x_{T}(t)+1-\sqrt{x_{T}^{2}+1}\right)$ and $\lambda_{l}(t)=\mu C G_{l}\left(x_{l}(t), x_{T}(t)\right)$ from (4) and (5), respectively. Solving these two equations along with (7), we obtain the utilization function for class $l$ traffic $G_{l}\left(x_{l}(t), x_{T}(t)\right)$ as:

$$
G_{l}\left(x_{l}(t), x_{T}(t)\right)=\frac{2 x_{l}(t)\left(\sqrt{x_{T}^{2}(t)+1}-x_{T}(t)\right)}{\sqrt{x_{T}^{2}(t)+1}-\left(x_{T}(t)-1\right)}
$$

Substituting (8) back into (5), we get:

$$
\begin{array}{r}
\dot{x}_{l}(t)=-\mu C\left[\frac{2 x_{l}(t)\left(\sqrt{x_{T}^{2}(t)+1}-x_{T}(t)\right)}{\sqrt{x_{T}^{2}(t)+1}-\left(x_{T}(t)-1\right)}\right]+\lambda_{l}(t) \\
\forall l=1,2, \ldots, S
\end{array}
$$

As a result, a node can be represented by a set of $S$ nonlinear equations of the form of Equation (9) describing the queue length dynamics of each class separately. The multiclass fluid flow model developed here represents the dynamics of a single node with Poisson input traffic and deterministic service times. Next we extend this model to study the behavior of a network.

\section{Hybrid Modeling of Multihop Wireless Networks}

In an $M$-node network, an arbitrary node $i$ is shown in Fig.1. At each node, there are $M-1$ possible packet classes based on different final destinations. We assume that packets are generated at node $i$ destined for node $j$ according to a Poisson process (which can be nonstationary) with mean rate $\gamma_{i}^{j}(t) . x_{i}^{j}(t)$ is the average number of packets at node $i$ buffer destined for node $j$. A routing variable $r_{i k}^{j}(t)$ denotes a zero/one indicator that equals to one if traffic from node $i$ destined to node $j$ is routed through node $k$ according to the specific routing scheme (e.g., DSR, AODV, etc.).

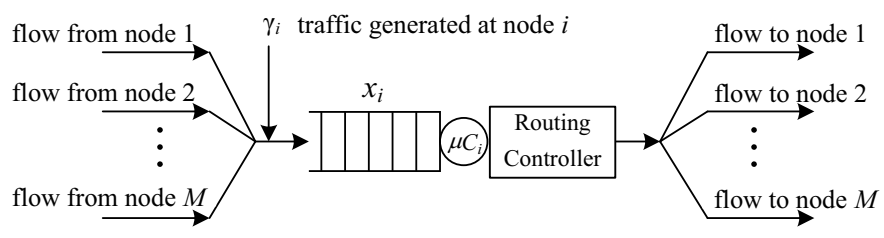

Fig. 1. An arbitrary node $i$ queueing model

To interconnect queues, it was suggested in [19], [20] that output from queueing system with deterministic service time should be treated as an delayed input to the next stage. Thus, the input to the next stage is basically nothing but a superposition of the delayed input streams from the nearby nodes plus any external arriving traffic $\gamma_{i}(t)$. Consider a two stage tandem queue model in Fig. 2(a)-(b), let $x_{i}(t), \lambda_{i}(t)$ and $G_{i}(t)$ be the average number in the system, total average arrival rate and average utilization at node $i$ respectively. Then $\lambda_{1}(t)=\gamma_{1}(t)$ is the arrival rate to the first queue, and $\mu C G_{1}(t)$ is the departure rate from the first queue. The departure rate then becomes the input of the second queue with a deterministic propagation delay of $D$, or $\lambda_{2}(t)=\mu C G_{1}(t-D)+\gamma_{2}(t)$. We can then write a set of differential equations to represent the rate of change in average number of packets at node 1 and 2, $x_{1}(t)$ and $x_{2}(t)$ for Fig. 2(a)-(b) as:

$$
\begin{aligned}
& \dot{x}_{1}(t)=-\mu C G_{1}(t)+\gamma_{1}(t) \\
& \dot{x}_{2}(t)=-\mu C G_{2}(t)+\gamma_{2}(t)+\mu C G_{1}(t-D)
\end{aligned}
$$

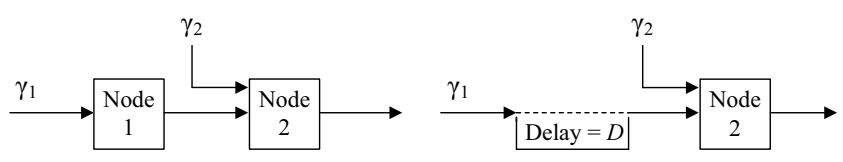

(a) Original System

(b) Equivalent Model

Fig. 2. A two-node deterministic service system with its equivalent model. 
Finally, the $M$ nodes network hybrid model is given by

$$
\begin{gathered}
\dot{x}_{i}^{j}(t)=-\mu C_{i} \frac{2 x_{i}^{j}(t)\left(\sqrt{x_{T}^{2}(t)+1}-x_{T}(t)\right)}{\sqrt{x_{T}^{2}(t)+1}-\left(x_{T}(t)-1\right)} \sum_{\substack{k=1 \\
k \neq i}}^{M} a_{i k}(t) r_{i k}^{j}(t) \\
+\sum_{\substack{l=1 \\
l \neq i, j}}^{M}\left[\mu C_{l} \frac{2 x_{l}^{j}(t-D)\left(\sqrt{x_{T}^{2}(t-D)+1}-x_{T}(t-D)\right)}{\sqrt{x_{T}^{2}(t-D)+1}-\left(x_{T}(t-D)-1\right)}\right. \\
\left.\left(a_{l i}(t) r_{l i}^{j}(t)\right)\right]+\gamma_{i}^{j}(t) \quad \forall i, j=1,2, \ldots, M
\end{gathered}
$$

The first term to the right of equal sign in (11) represents class $j$ traffic flow out of node $i$, while the second term shows the class $j$ traffic flow routed into node $i$ from other neighboring nodes. The last term denotes the class $j$ traffic generated by node $i$. This differential equations based model can be solved via numerical integration techniques (e.g., Runge Kutta).

\section{E. Additional Performance Measures}

In this section, we show that one can use the proposed fluid flow modeling approach to estimate other performance metrics, besides the average queue length in buffer. For the sake of brevity, we discuss the estimation of the end-to-end delay here only. In general, the queuing delay is considered as the main factor of node's delay, which depends on the queue size. According to Little's theorem, the average number in the system is equivalent to the product of the average arrival rate and the average time in the system. If $x$ denotes the average number in the system, $\lambda$ is the average arrival rate and $W$ is the average waiting time, then $x=\lambda W$. With the assumption of constant arrival rate over a small step, the change in average waiting time can be related to the rate of change in average number in the system $\dot{W}=\dot{x} / \lambda$. We now consider a path $P$ of $j-1$ hops from source node 1 to destination node $j$ (class $j$ traffic), given by $(1,2),(2,3) \ldots(j-1, j)$, where $(i, i+1)$ represents a link on the path, for $\forall i=1,2 \ldots j-1$. The average node queuing delay at node $i$ for class $j$ traffic on link $(i, i+1)$ is denoted by $W_{i}^{j}(t)$. Since we are more interested in the time dependent behavior, the rate of change of $W_{i}^{j}(t)$ is given by:

$$
\dot{W}_{i}^{j}(t)=\sum_{i=1}^{j-1} \frac{\dot{x}_{i}^{j}(t)}{\lambda_{i}^{j}(t)} \quad \forall i=1,2, \ldots, j-1
$$

where

$$
\begin{aligned}
\lambda_{i}^{j}(t) & =\sum_{\substack{l=1 \\
l \neq i, j}}^{M}\left[\frac{2 x_{l}^{j}(t-D)\left(\sqrt{x_{T}^{2}(t-D)+1}-x_{T}(t-D)\right)}{\sqrt{x_{T}^{2}(t-D)+1}-\left(x_{T}(t-D)-1\right)}\right. \\
& \left.\mu C_{l}\left(a_{l i}(t) r_{l i}^{j}(t)\right)\right]+\gamma_{i}^{j}(t)
\end{aligned}
$$

In general, the link propagation delay $T_{i, i+1}$ is considered fixed and almost equal for each hop on the path. If necessary, it could be added to the node queuing delay. As a result, the end-to-end delay of path $P$ with $j-1$ hops can be written as:

$$
D_{P}(t)=\sum_{i=1}^{j-1} W_{i}^{j}(t)+(j-1) T_{i, i+1}
$$

\section{F. Hybrid Modeling Algorithm}

At the end of this section, we summarize our hybrid modeling procedure as the following algorithm to estimate the time dependent performance metrics of multihop wireless networks over the desired time interval $\left[t_{0}, t_{f}\right]$.

1. Initialization: set the current time $t=t_{0}$ and the time step $\Delta t$. Let the initial variable $x_{i}^{j}(t)=x_{i}^{j}\left(t_{0}\right)$, which is the node $i$ occupancy by the packets destined for node $j$. Also, specify network parameters including the link capacity $C$, the packet length $1 / \mu$ and the packet service duration $D$.

2. Determine the traffic routes $r_{i k}^{j}(t)$ according to the routing protocol and the adjacency matrix $A(t)$ at time $t$. Also, update the mean rate $\gamma_{i}^{j}(t)$ of each node's offered traffic.

3. Numerically solve the differential equations (11) and get the new $x_{i}^{j}(t+\Delta t)$ at the end of the time interval $[t, t+\Delta t]$, which then becomes the initial condition for the next time interval $[t+\Delta t, t+2 \Delta t]$.

4. To estimate the end-to-end delay $D_{P}(t+\Delta t)$, sum up the link propagation delays $T_{i, i+1}$ with the node queuing delays $W_{i}^{j}(t+\Delta t)$ along the path $P$. Here, $W_{i}^{j}(t+\Delta t)$ is obtained by numerically solving the differential equation (12) over the time interval $[t, t+\Delta t]$ and then it turns out to be the initial condition for the next time interval $[t+\Delta t, t+2 \Delta t]$.

5. Increment time $t=t+\Delta t$. If $t<t_{f}$, go back to step 2; otherwise terminate.

\section{Hybrid Model VAlidation By discRete EVEnT SIMULATION}

\section{A. Numerical Experiments}

In this section, our model is validated by comparing with an equivalent discrete event simulation model built with OPNET [21]. In the OPNET simulation model, each queue of the node is configured as a FIFO queues with infinite buffer size and each class traffic is buffered at its corresponding subqueue. The discrete event simulation results are the ensemble average of 5000 replications with $99 \%$ confidence intervals by using the nonstationary simulation methodology.

As illustrated in Fig. 3 (a)-(f), we study a simple simulation scenario of three nodes with pre-determined connectivity changes between nodes at each time interval. Minimum hop routing is applied in this setup. When the direct link is no longer available, traffic must be rerouted through relay nodes and uses some available portion of the shared link capacity. In both the analytical model and the simulation, we set the link capacity for all nodes as $C_{i}=10^{4} \mathrm{bps}$ with fixed packet length $1 / \mu=1250$ bytes, so that the service rate $\mu C_{i}$ is normalized as $1 \mathrm{pkt} / \mathrm{s}$, which is the inverse of the deterministic service time $D$. Each node generates Poisson traffic with the mean rate $(\mathrm{pkt} / \mathrm{s})$ of $\gamma_{1}^{2}=0.16, \gamma_{1}^{3}=0.2, \gamma_{2}^{1}=0.16, \gamma_{2}^{3}=0.2, \gamma_{3}^{1}=$ $0.16, \gamma_{3}^{2}=0.2$. The link connectivity $a_{i j}$ is set to be binary and the link propagation delay is assumed to be 0.01 seconds.

Fig. 4 shows the effect of topology change on the average packet number and end-to-end delay for the traffic at node 1 


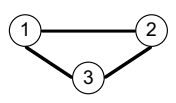

(a) $t<100$

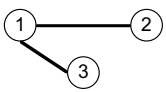

(d) $300<=t<400$

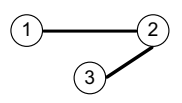

(b) $100<=t<200$

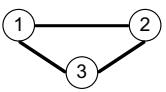

(e) $400<=t<500$

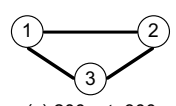

(c) $200<=t<300$

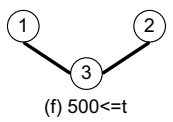

Fig. 3. Three node network connectivity scenario.

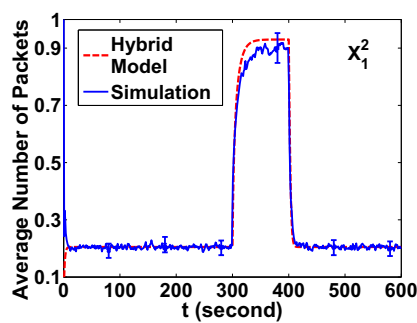

(a)

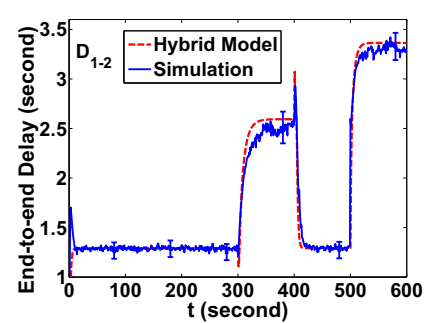

(b)
Fig. 4. Dynamic behavior of the traffic destined for node 2 at node 1 buffer.

buffer and destined for node 2, as computed via our hybrid model and nonstationary simulation. For the time interval $t<$ $100 \mathrm{sec}$, the network is fully connected. All nodes go through an initial transient period and then reach the steady state. For time $100 \leq t<200 \mathrm{sec}$, link between node 1 and 3 breaks, so that their traffic has to be re-routed through the relay node 2 . But the packets $x_{1}^{2}$ buffered in source node 1 are not affected. Then, the broken link 1-3 is restored during the time interval $200 \leq t<300 \mathrm{sec}$, and all nodes recover themselves to the steady state. During the time interval $300 \leq t<400 \mathrm{sec}$, the link between node 2 and 3 breaks, leading to the traffic from node 2 and 3 re-routed through node 1 . Thereby, more packets $x_{1}^{2}$ are buffered in node 1 . Due to higher server utilization, the queueing delay in node 1 is increased so that the end-toend delay $D_{1-2}$ from node 1 destined for node 2 get raised. Starting from the time $500 \leq t$ sec, the link between node 1 and 2 is broken and the traffic in $x_{1}^{2}$ has to go through the relay node 3 to reach the destination. Thus, $D_{1-2}$ experiences the delay of two hops including the propagation delay of link 1-3 and 3-2 as well as the queuing delay at node 1 and 3. The behavior of other nodes and traffic streams are similar and not shown here for the purpose of brevity. From Fig. 4, we can see that the hybrid model is fairly accurate and matches well with the discrete event simulation results.

\section{B. Computational Scalability}

In order to show the hybrid model is a more scalable tool than nonstationary simulation, we give a brief analysis on the computation time complexity of the hybrid model followed by numerical results comparison. For an $M$-node network, each node has $M-1$ possible destination-based traffic classes, and hence the hybrid model generates $M(M-1)$ differential equations. In [22], by using $p$-th order explicit Runge-Kutta algorithm with maximum error $e^{-\alpha}$, the upper bound of the number of arithmetic operations required to solve $n$ differential equations is given as $C(n, p, \alpha)$. With a pre-defined value of $p$ and $\alpha$, the upper bound function $C(n)$ increases linearly with $n$ according to its expression in [22]. Therefore, for an $M$-node network, the upper bound of the computation time complexity of the hybrid model is $O(M(M-1))$.

Then we conduct numerical experiments on the sample networks to quantitavely evaluate the computational complexity of our hybrid modeling approach and compare it with nonstationary simulation. In each sample network with full mesh links, all links switch between on/off randomly. The computation time of the hybrid model solved by Matlab is compared with nonstationary simulation [10] with 5000 independent runs from OPNET in Table I; both are implemented on a PC with a Intel T7400 2.16 GHz duo-core processor and 2GB memory. All the numerical queue length results from the hybrid model are within $99 \%$ confidence interval of the simulation results. As seen from the table, the simulation time seems to grow exponentially, which is actually a complex function of number of nodes, amount of traffic, topology changes and accuracy desired. For the hybrid model implemented in MATLAB, the computation time is roughly proportional to the number of the fluid flow differential equations $M(M-1)$, as expected from our analysis above.

TABLE I

COMPUTATION TIME COMPARISON

\begin{tabular}{|c|c|c|c|}
\hline \# Nodes & \# Diff. Equations & Simulation $(\mathrm{sec})$ & Hybrid Model $(\mathrm{sec})$ \\
\hline 3 & 6 & 142.3 & $\mathbf{0 . 2 4}$ \\
\hline 4 & 12 & 1235.6 & $\mathbf{0 . 5 2}$ \\
\hline 5 & 20 & 12872.8 & $\mathbf{0 . 8 3}$ \\
\hline 13 & 156 & 402931.6 & $\mathbf{7 . 0 2}$ \\
\hline
\end{tabular}

\section{Performance Evaluation via Hybrid Model}

\section{A. Nodes Mobility Impact}

We consider the impact of nodes mobility on the performance of multihop wireless networks by using the random waypoint mobility (RWM) model for node movement. Stochastic properties of the RWM model were studied in [18] and show that the connectivity of two nodes is memoryless, which means the future connectivity of two nodes depends only on the current state of the connectivity. Hence, the link connectivity can be modeled as a two-state Markov process with on-off (connected-disconnected) transition, and both link on and off durations follow exponential distributions [18]. This model can represent the average link stability statistics of RWM model without a long warm-up simulation period.

A five node network is set up with mean link on lifetime $T_{\text {on }}=50 \mathrm{sec}$ and mean link off lifetime $T_{\text {off }}=20 \mathrm{sec}$. All links are assumed to have the same $T_{\text {on }}$ and $T_{\text {off }}$. In the experiments, the network parameters are kept the same as three node network before. The mean rate (pkt/s) of the traffic generated by each node is $\gamma_{1}^{3}=0.22, \gamma_{1}^{5}=0.28, \gamma_{2}^{5}=$ $0.12, \gamma_{3}^{5}=0.21, \gamma_{4}^{5}=0.16$. We illustrate typical dynamic network performance by ploting the traffic destined for node 5 at node 1, as seen in Fig. 5. We show four snapshots of 

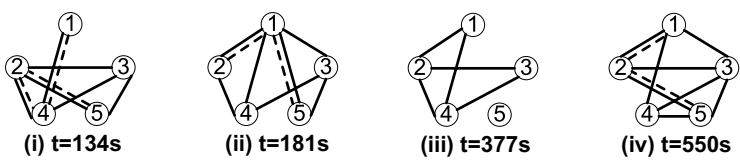

(a) Sample topologies

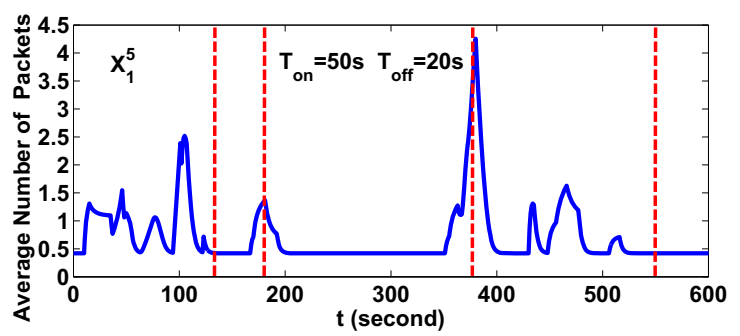

(b) Average number of packets destined for node 5 at node 1 buffer

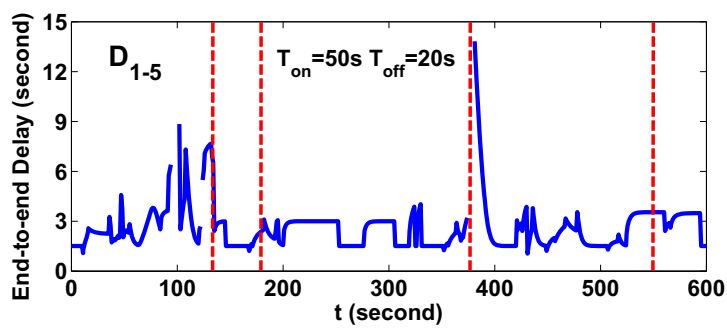

(c) Average end-to-end delay from node 1 to node 5

Fig. 5. Dynamic Behavior of the traffic destined for node 5 at node 1 buffer with node mobility model $T_{o n}=50 \mathrm{~s}, T_{o f f}=20 \mathrm{~s}$.

the network topology at different times in Fig. 5(a) and mark all the routes of class 5 (destined for node 5) traffic from node 1 by dot lines in the topologies. Then, we associate the performance results with each topology by aligning them at the same time instant in Fig. 5(b)(c). The results conform with the facts that when the direct link breaks as topologies (i) and (iv) of Fig. 5(a), the traffic has to go through multiple hops to the destination resulting in longer end-to-end delay, while the average packet number of this traffic in source node buffer remains the same value as direct link exists. Once destination node 5 is isolated from the network as in topology (iii), the instantaneous end-to-end delay $D_{1-5}$ becomes infinitely large, and the packets have to be queued up in the buffer of source node 1 . When node 1 helps forward packets of other nodes to destination node 5 at topology (ii), more packets in the buffer causes longer queueing delay in node 1 .

Next we increased the average link off lifetime $T_{\text {off }}=40 \mathrm{~s}$ and decreased the link on lifetime $T_{o n}=30 \mathrm{~s}$ for each pair of nodes to observe a higher level of nonstationarity, since each node will have less overall connectivity and is more likely to redirect traffic. All the other network parameters remain the same as before. Typical results are given in Fig. 6, for traffic at node 1 destined for node 5. When the effect of long average link off lifetime starts to set in, it becomes more difficult for each node to find any intermediate node to relay the traffic. When the source node cannot find any alternative path to reroute the traffic, the instantaneous end-to-end delay becomes infinitely large and appears to be disconnected, and

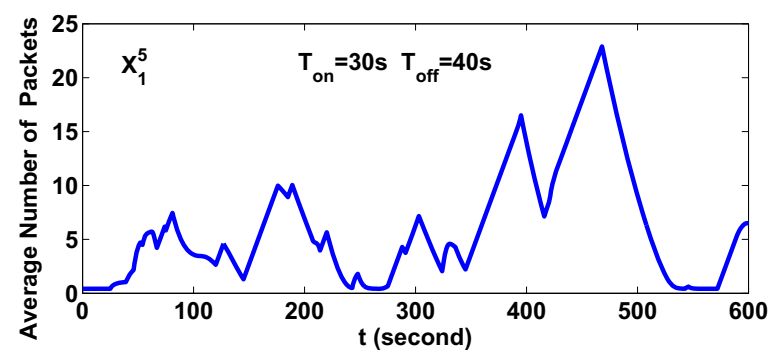

(a) Average number of packets destined for node 5 at node 1 buffer

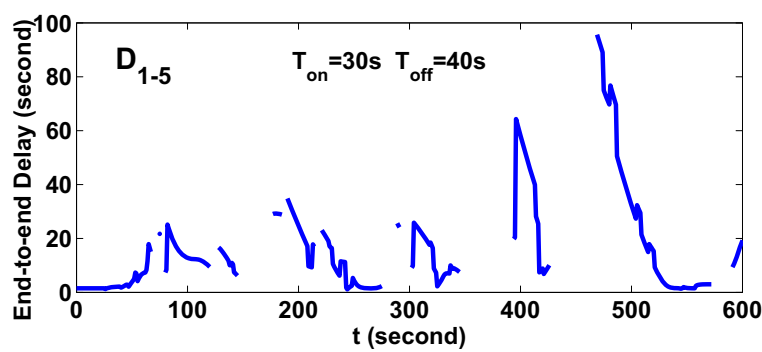

(b) Average end-to-end delay from node 1 to node 5

Fig. 6. Dynamic Behavior of the traffic destined for node 5 at node 1 buffer with node mobility model $T_{o n}=30 \mathrm{~s}, T_{o f f}=40 \mathrm{~s}$.

meanwhile the packets are accumulated linearly.

The concept of two-state Markov model can represent various mobility scenarios by using different combinations of $T_{o n}$ and $T_{o f f}$. For example, the random waypoint mobility of the wireless nodes with smaller radio range can be implicitly represented by relatively larger $T_{o f f}$. Alternately, in a random waypoint group mobility, if two nodes are from the same group, the average $T_{o n}$ of the link between these two nodes should be longer, since they tend to be moving with a comparable speed in a similar direction.

\section{B. Traffic Load and Link Quality Impact}

In wireless networks, communication may exhibit diverse link quality in terms of the error rate and data rate as discussed earlier. Measurement data in [16] shows that as long as the link exists, the inter-node distance has little predictive value for error rate. Instead, the error rate is probably due to obstacles, multi-path fading and interference. In other words, up to a certain inter-node distance, the error rate is dominated by obstacles, geometry and interference. Thus, instead of $a_{i j}=1$, decimal numbers $a_{i j}$ are randomly assigned to all links in the network within certain range to indicate the error effect in certain environment. Here we set up a 30 node network with full mesh traffic, and focus on a sample traffic destined for node 30 at node 1 buffer. All the network parameters remain the same as before, except for lightly loaded traffic and probabilistic link connectivity. All three scenarios in Fig. 7 use the same mobility model $\left(T_{o n}, T_{o f f}\right)=(50 s, 20 s)$ and the minimumhop routing $r_{i k}^{j}$. We first evaluate the impact of traffic load in a "higher" link quality environment $a_{i j} \in\left[\begin{array}{ll}0.95 & 1\end{array}\right]$. When the offered traffic of each node increases twice from $\gamma_{i}^{j}=4 \times 10^{-3}$ $\mathrm{pkt} / \mathrm{s}$ to $8 \times 10^{-3} \mathrm{pkt} / \mathrm{s}$, the average queue length $x_{1}^{30}$ almost doubles, but the end-to-end delay $D_{1-30}$ rises slightly. This 


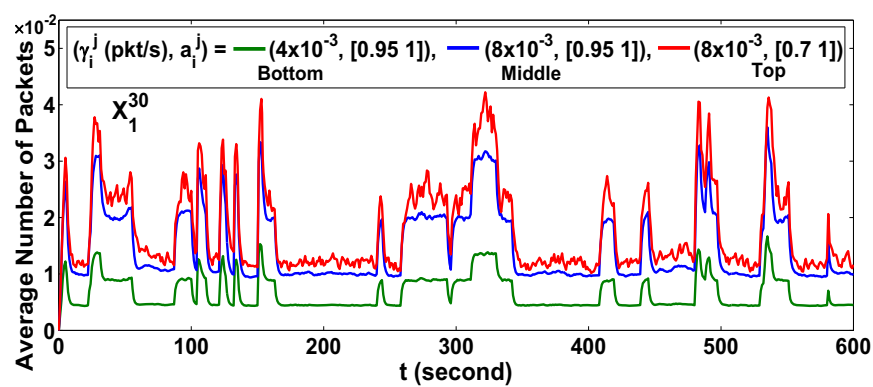

(a) Average number of packets destined for node 30 at node 1 buffer

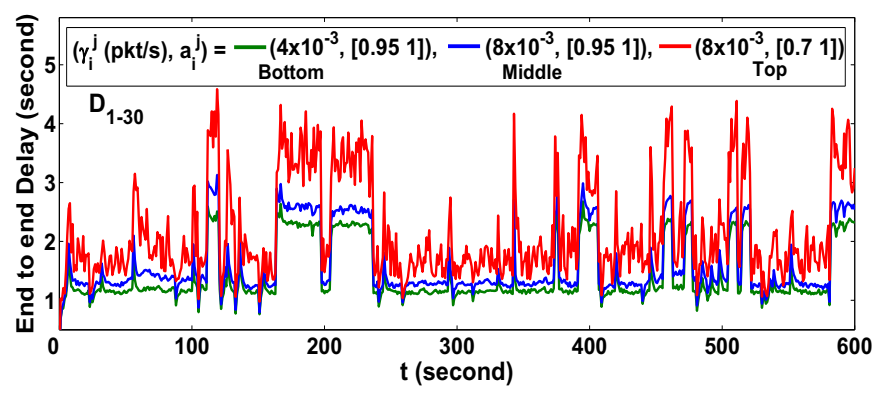

(b) Average end-to-end delay from node 1 to node 30

Fig. 7. Dynamic Behavior of the traffic destined for node 30 at node 1 buffer under various offered loads and link quality conditions with mobility $T_{\text {on }}=50 \mathrm{~s}, T_{\text {off }}=20 \mathrm{~s}$.

phenomena are conform to the $M / D / 1$ steady state Equation (7) and Little's theorem with light traffic $\lambda$. Then we fix the traffic load at $\gamma_{i}^{j}=8 \times 10^{-3} \mathrm{pkt} / \mathrm{s}$ and evaluate the network performance in "lower quality" wireless environment with link connectivity $a_{i j}$ ranged in [0.7 1]. Since a lower link quality inevitably reduces wireless link capacity, more packets have to be buffered at each node and the queuing delay is prolonged accordingly. In addition, these two numerical ranges reflect the fact that "lower quality" wireless channel typically has larger variance than "higher quality" one; thereby the network performance behaves with larger fluctuations.

As we know, the steady-state simulation typically eliminates the information gathered during the transient period to avoid initialization bias and mostly depends on the steady-state values. The results from Fig. 5-7 illustrate the application of the hybrid model to the study of nonstationary conditions and its capability to capture the network transient behavior.

\section{CONCLUSiON}

In this paper, we develop a hybrid model for nonstationary queuing analysis of multihop wireless network with deterministic service times. The proposed model consists of two components: the network topology modeling and the time dependent queuing behavior modeling. Numerical results for a sample network via the proposed model are validated by simulations. The proposed hybrid approach is shown to be much more computationally efficient than the equivalent discrete event simulation. Furthermore, we apply this hybrid model to examine the impact of node mobility, offered traffic load, and wireless link quality on network performance. Without sacrificing a large amount of computational resources, we believe the hybrid model to be an alternative to provide flexibility in modeling complex networks with nonstationary effects. Future work is to model the effect of MAC layer contention in dense or heavily loaded multihop wireless networks and integrate it into the hybrid model.

\section{REFERENCES}

[1] I. Akyildiz and X. Wang, "A survey on wireless mesh networks," Communications Magazine, IEEE, vol. 43, no. 9, pp. S23 - S30, 2005.

[2] H. Moustafa and Y. Zhang, Vehicular networks, Techniques, Standards, and Applications. CRC Press, 2009.

[3] I. F. Akyildiz, W. Su, Y. Sankarasubramaniam, and E. Cayirci, "Wireless sensor networks: a survey," Computer Networks, vol. 38, pp. 393-422, 2002.

[4] I. Chlamtac, M. Conti, and J. Liu, "Mobile ad hoc networking: Imperatives and challenges," Ad Hoc Networks, vol. 1, no. 1, pp. 13-64, 2003.

[5] B. N. J. Banks, J. Carson and D. Nicol, Discrete Event System Simulation, 4th ed. Prentice-Hall, 2005.

[6] D. Tipper, Y. Qian, and X. Hou, "Modeling the time varying behavior of mobile ad-hoc networks," in Proc. of 7th ACM International Symposium on Modeling, Analysis and Simulation of Wireless and Mobile Systems, Oct. 2004, pp. 12-19.

[7] W. Lovegrove, J. Hammond, and D. Tipper, "Simulation methods for studying nonstationary behavior of computer networks," IEEE Journal on Selected Areas in Communications, vol. 8, no. 9, pp. 1696-1708, 1990.

[8] P. V. Meighem, Performance Analysis of Communications Networks and Systems. Cambridge University Press, 2006.

[9] D. Tipper and M. K. Sundareshan, "Numerical methods for modeling computer networks under nonstationary conditions," IEEE Journal on Selected Areas in Communications, vol. 8, no. 9, pp. 1682-1695, 1990.

[10] W. Wang, D. Tipper, and S. Banerjee, "A simple approximation for modeling nonstationary queues," in Proc. of IEEE INFOCOM, Mar. 1996, pp. 255-262.

[11] K. Xu, S. Tipmongkonsilp, D. Tipper, P. Krishnamurthy, and Y. Qian, "A time dependent performance model for multihop wireless networks with CBR traffic," in Proc. of IEEE International Performance Computing and Communications Conference (IPCCC), Dec. 2010, pp. $271-280$.

[12] H. Kim and J. Hou, "How good is fluid model-based simulation for simulating IEEE 802.11 operated WLANs," in Proc. of Communication Networks and Distributed Systems Modeling and Simulation Conference (CNDS), Jan. 2003.

[13] P. M. Atilla Eryilmaz and A. Ozdaglar, "A fluid-flow model for backlogbased CSMA policies," in Proc. of Annual International ICST Conference on Wireless Internet (WICON), Jan. 2008, pp. 77:1-77:9.

[14] V. Kanodia, C. Li, A. Sabharwal, B. Sadeghi, and E. Knightly, "Distributed multi-hop scheduling and medium access with delay and throughput constraints," in Proc. of ACM MobiCom, July 2001, pp. 200209.

[15] A. Veres, A. Campbell, M. Barry, and L.-H. Sun, "Supporting service differentiation in wireless packet networks using distributed control," IEEE Journal on Selected Areas in Communications, vol. 19, no. 10, pp. 2081-2093, 2001

[16] D. Aguayo, J. Bicket, S. Biswas, G. Judd, and R. Morris, "Linklevel measurements from an $802.11 \mathrm{~b}$ mesh network," in Proc. of ACM SIGCOMM, Sep. 2004, pp. 121-132.

[17] T. Camp, J. Boleng, and V. Davies, "A survey of mobility models for ad hoc network research," Wireless Communication \& Mobile Computing (WCMC), vol. 2, no. 5, pp. 483-502, 2002.

[18] S. K. Hwang and D. S. Kim, "Markov model of link connectivity in mobile ad hoc networks," Telecommunication System, vol. 34, no. 1-2, pp. 51-58, 2007.

[19] M. J. Neely, "Exact queueing analysis of discrete time tandems with arbitrary arrival processes," in Proc. of IEEE ICC, 2004, pp. 2212-2225.

[20] M. Neely, "Equivalent models for queueing analysis of deterministic service time tree networks," IEEE Transaction on Information Theory, vol. 51, no. 10, pp. 3576-3584, 2005.

[21] OPNET Simulaiton Tool, http://www.opnet.com/.

[22] A. Werschulz, "Computational complexity of one-step methods for systems of differential equations," Mathematics of Computation, vol. 34, no. 149, pp. 155-174, 1980. 